\title{
Penicillin and Beyond: Evolution, Protein Fold, Multimodular Polypeptides, and Multiprotein Complexes
}

\author{
JEAN-MARIE GHUYSEN, PAULETTE CHARLIER, JACQUES COYETTE, COLETTE DUEZ, \\ EVELINE FONZÉ, CLAUDINE FRAIPONT, COLETTE GOFFIN, BERNARD JORIS, and \\ MARTINE NGUYEN-DISTÈCHE
}

\begin{abstract}
As the protein sequence and structure databases expand, the relationships between proteins, the notion of protein superfamily, and the driving forces of evolution are better understood. Key steps of the synthesis of the bacterial cell wall peptidoglycan are revisited in light of these advances. The reactions through which the D-alanyl-D-alanine depeptide is formed, utilized, and hydrolyzed and the sites of action of the glycopeptide and $\beta$-lactam antibiotics illustrate the concept according to which new enzyme functions evolve as a result of tinkering of existing proteins. This occurs by the acquisition of local structural changes, the fusion into multimodular polypeptides, and the association into multiprotein complexes.
\end{abstract}

\section{INTRODUCTION}

$\mathbf{T}$ HE TRANSLATION OF GENETIC INFORMATION into biological activity is achieved by the conversion of a newly synthesized polypeptide chain into a compact, correctly folded protein. ${ }^{8}$ The folding code is still far from being understood. Nascent proteins fold rapidly, in seconds or less, in spite of the fact that the time that would be needed to search all potentially accessible conformations is astronomical. The solution to this paradox is that an essential step in protein folding is the formation of a "molten globule." This species lacks the persistent tertiary interactions characteristic of the native state, but it already possesses extensive secondary structures that are major elements of the native topology. From this intermediate, the remaining search for correct folding is only over a limited conformational area. Moreover, the cells possess many factors that assist folding and minimize and/or correct misfolding events.

The distribution of amino acid residue types along the polypeptide chain is a major determinant of secondary and tertiary structures. Yet, the number of distinct folds adopted by the proteins is limited. Proteins having $25 \%$, or more, of their sequences in common adopt the same folded structures. But, at the same time, an increasing number of proteins are being revealed that have similar folds and statistically insignificant similarities. ${ }^{32}$ Hence, proteins unrelated in sequence and function may diverge from a common protein ancestor while retaining the same basic polypeptide fold. It has been reported ${ }^{32}$ that 2511 polypeptide chains cluster into 212 amino acid sequence families ( $25 \%$, or more, identities) and into only 80 single domain polypeptide fold families. In consequence, a classification has been considered that extends the sequence-based superfamilies to include proteins with similar three-dimensional structures but no sequence similarity. One may also note that nine superfolds dominate the protein database, representing more than $30 \%$ of all determined structures. ${ }^{32}$ Often, evolution obscures the function.

The pathway of the bacterial cell wall peptidoglycan synthesis shown in Figure 1 is that of Escherichia coli. It applies to all bacteria possessing a wall peptidoglycan. From the MurA UDP-Nacetylglucosamine enolpyruvate transferase, ${ }^{4}$ which catalyzes the first committed step of the pathway in the cytoplasm, to the penicillin-binding proteins that assemble the polymer from the disaccharide-pentapeptide-lipid II intermediate on the outer face of the membrane, all the reactions are bacteria-specific. The lipid II intermediate is a $\beta$-1, 4-linked $N$-acetylmuramyl- $N$-acetylglucosamine disaccharide, the $N$-acetylmuramic acid of which is substituted by a D-alanyl-D-alanine-terminated pentapeptide via a D-lactyl-L-alanine amide bond and the C-1 atom is attached to the intracellular end of a transmembrane undecaprenyl lipid carrier via a pyrophosphate. Lipid II is a key intermediate. It is, at the same 


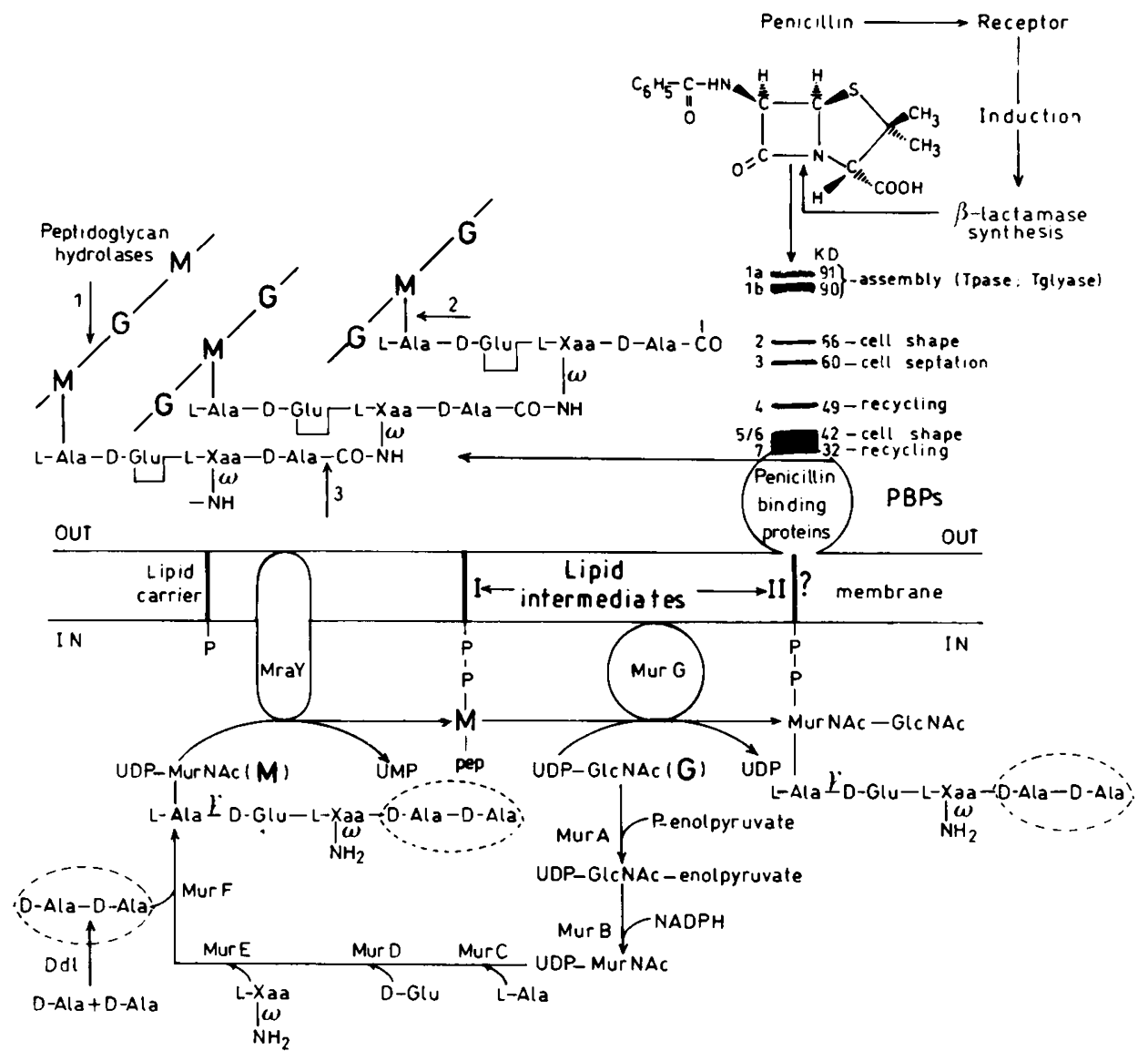

FIG. 1. Wall peptidoglycan synthesis pathway. The PBP pattern shown is that of $E$. coli in which case the diamino acid residue L-Xaa is meso-diaminopimelic acid. G, $N$-acetylglucosamine; $\mathrm{M}, N$-acetylmuramic acid; Tpase, transpeptidase; Tglyase, transglycosylase. The sites of cleavage of peptidoglycan hydrolases are shown: $1, N$-acetylmuramidase; $2, N$-acetylmuramoyl-L-alanine amidase; 3 , endopeptidase. The PBPs are inactivated by penicillin. Penicillin is hydrolyzed by the $\beta$-lactamases. In some bacteria, $\beta$-lactamase synthesis inducibility is mediated by a receptor (see Fig. 7). The PBPs, the majority of the $\beta$-lactamases, and the BIaR-type penicillin receptors belong to the superfamily of penicilloyl serine transferases.

time, the product of the "cytosolic" stage and the substrate of the "wall" stage of the synthesis.

The Ddl D-Ala-D-Ala ligase, the low-molecular-mass penicill-inbinding proteins (PBPs), and the $\beta$-lactamases have one single catalytic function. Some peptidoglycan hydrolases, the BlaR-type penicillin receptors, and the high-molecular-mass PBPs are multimodular polypeptides. Sets of PBPs and nonpenicillin-binding proteins associate into multiprotein complexes (not shown in Fig. 1) and form morphogenetic networks. These systems of increasing complexity are examined successively. With few exceptions, references are made only to papers published from 1993 to 1995.

\section{D-ALA-D-ALA AND D-ALA-D-LACTATE (ATP:ADP + $\mathbf{P}_{\mathrm{i}}$ ) LIGASES}

In $E$. coli, the lipid II intermediate is formed by the sequential addition of L-Ala, D-Glu, meso- $\mathrm{A}_{2} \mathrm{pm}$, and a preformed D-Ala-D-Ala to UDP- $N$-acetylmuramic acid by the MurC, MurD, MurE, and MurF adding enzymes, respectively (Fig. 1). The D-Ala-D-Ala dipeptide is synthesized by the Ddl adding en- zyme. The MraY and MurG transferases catalyze the attachment of the $\mathrm{N}$-acetylmuramyl pentapeptide to the lipid carrier and the subsequent addition of $\mathrm{N}$-acetylglucosamine, respectively.

The synthesis of the D-Ala-D-Ala dipeptide (Fig. 2) begins with the attachment of a first D-alanine residue on the $\gamma$-phosphate of adenosine triphosphate (ATP) to yield an acyl phosphate, followed by attack by the amino group of the second D-alanine residue to produce a tetrahedral intermediate, which then eliminates the phosphate group to give the D-Ala-D-Ala dipeptide. The DdlB D-Ala-D-Ala ligase of $E$. coli is made of three domains, ${ }^{14}$ each folded around a 4 - to 6 -stranded $\beta$-sheet core, and the ATP-binding site is sandwiched between the $\beta$-sheets of the carboxy-terminal and central domains. A helix dipole and the hydrogen-bonded catalytic triad E15, S150, and Y216 assist binding and deprotonation steps.

The insertion of lipid-transported, but as yet non-cross-linked disaccharide pentapeptide units, in the growing wall peptidoglycan must be achieved by transglycosylation at the level of the glycan chains and by transpeptidation at the level of the peptide chains if the process is to yield an insoluble network. As discussed below, peptide cross-linking by transpeptidation 


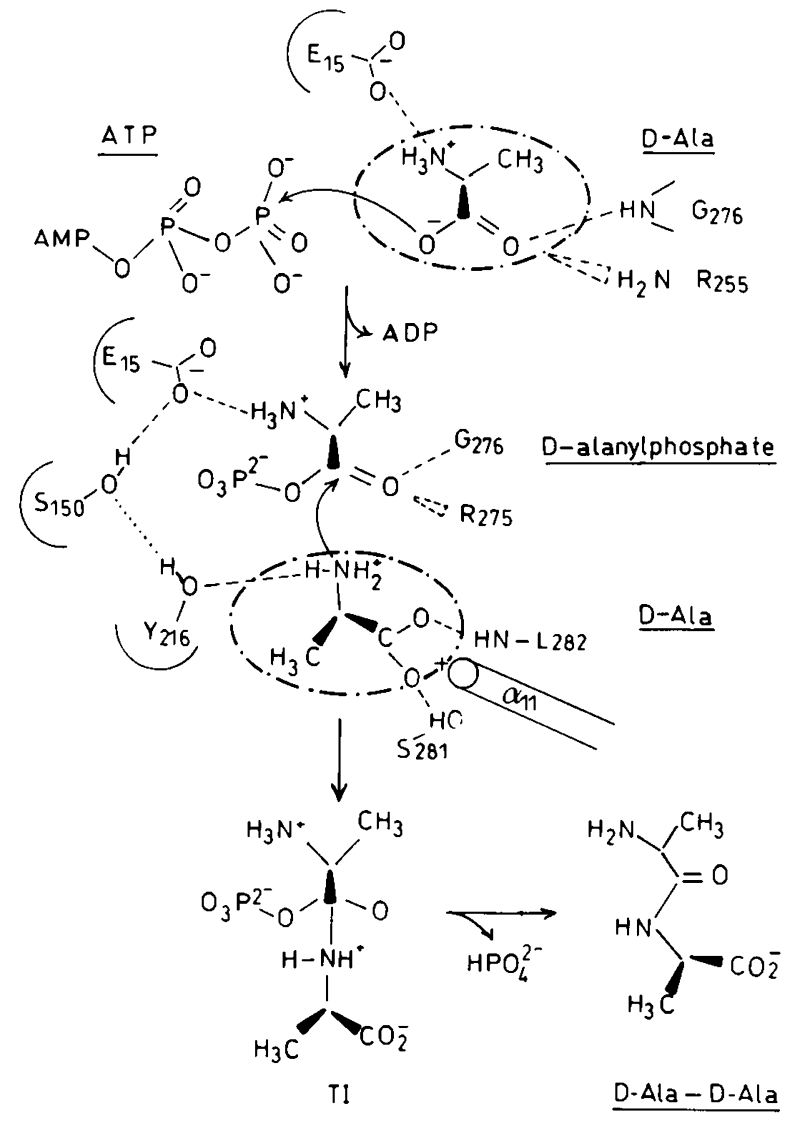

FIG. 2. Synthesis of the D-Ala-D-Ala dipeptide by the Ddl (ATP:APP $+\mathrm{P}_{\mathrm{i}}$ ) ligase.

is a reaction in which the carboxy-terminal D-alanyl-D-alanine moiety of a pentapeptide precursor serves as carbonyl donor. The glycopeptide antibiotics bind tightly to the dipeptide moiety of the lipid-transported disaccharide-pentapeptide precursors, preventing cross-linking.

Resistance to the glycopeptide vancomycin in enterococci results from changes in the peptidoglycan biosynthetic pathway. ${ }^{37}$ In VanA and VanB strains, the dipeptide D-Ala-D-Ala of the peptidoglycan precursor is replaced by the depsipeptide D-Ala-D-lactate. This change does not limit the activity of the transpeptidase that catalyzes cross-linking, but it results in, at least, a 1000-fold decreased binding affinity of vancomycin for the peptidoglycan precursor. The D-Ala-D-Ala and D-Ala-D-lactate ligases have $30 \%$ of their sequences in common. ${ }^{14}$ Hence, conversion of one ligase into the other requires a great extent of local changes but it does not alter the fold topology. The crutial E15 and S150 are conserved, but notable differences also occur in the active site, the most significant one being the substitution $\mathrm{Y} 216 \rightarrow \mathrm{K}$.

The DdlB D-Ala-D-Ala ligase and the $\gamma$-glutamyl cysteineglycine ligase (or glutathione synthetase) couple activation of an acyl group and hydrolysis of ATP into ADP and $P_{i}$ to provide the thermodynamic driving force for peptide bond synthesis. The two synthetases perform different functions (glutathione is the major determinant of the oxidation-reduction state of the cells). They lack amino acid sequence similarity
(10\% identities). Yet they show a remarkable fold similarity. ${ }^{15}$ Their common signature fold and catalytic site may be characteristics of a particular superfamily of ADP-forming peptide synthetases. The MurC, MurD, MurE, and MurF ligases and the $\gamma$-glutamic acid-cysteine ligase (the reaction product of which is the substrate of the glutathione synthetase) also perform peptide bond formation with concomitant hydrolysis of ATP into ADP and $P_{i}$. They might be other members of the same superfamily.

\section{MONOFUNCTIONAL PENICILLIN-BINDING PROTEINS AND $\beta$-LACTAMASES}

Serine-assisted transpeptidation between a D-Ala-D-Ala-terminated pentapeptide precursor acting as carbonyl donor and the $\omega$-amino group of the $\mathrm{L}$-Xaa residue of another peptide acting as amino acceptor does not require an input of energy and, therefore, can result in peptide bond formation at exocellular sites where ATP is not available.

The transpeptidation reaction requires a precise proton abstraction-donation (Fig. 3). In step 1, the C-terminal D-Ala-DAla dipeptide moiety of a pentapeptide precursor must bind to the active site of the enzyme in a position that allows the proton of the $\gamma \mathrm{OH}$ of the active-site serine $\left(\mathrm{S}^{*}\right)$ to be abstracted, the activated $\mathrm{O} \gamma \mathrm{S}^{*}$ to attack the carbonyl of the D-Ala-CONHD-Ala scissile bond, and the abstracted proton to be back-donated to the adjacent nitrogen atom. In step 2 , the serine $\left(\mathrm{S}^{*}\right)$ ester-linked peptidyl enzyme must adopt a conformation that allows the proton of the $\omega$-amino group of the L-Xaa residue of another peptide to be abstracted, the activated $\ddot{\mathrm{N}} \mathrm{H}$ to attack the carbonyl of the ester bond, and the abstracted proton to be back-donated to the $\mathrm{O} \gamma \mathrm{S}^{*}$ atom. Backbone amino groups of the enzyme cavity (denoted E-NH in Fig. 3) polarize the carbonyl of the D-Ala-D-Ala peptide bond in step 1 and the carbonyl of the peptidyl enzyme ester bond in step 2 .

Because the dipeptide D-Ala-D-Ala (in its extended conformation) and penicillin are nearly isosteric, the transpeptidase also reacts with penicillin. But because the scissile $\beta$-lactam amide bond is endocyclic, the serine $\left(\mathrm{S}^{*}\right)$ ester-linked penicilloyl enzyme is very long lived. The transpeptidase is inactivated and behaves as a PBP (Fig. 4).

An evolutionary scenario has been proposed ${ }^{18}$ through which acquisition of new functions from a putative DD-transpeptidase/PBP ancestor is achieved by local changes (Figs. 3 and 4). Catalyzed hydrolysis of the ester bond of the peptidyl enzyme with conservation of the inertness of the penicilloyl enzyme gave rise to the monofunctional DD-carboxypeptidases/PBPs. They may control the extent of peptidoglycan cross-linking. Conversely, catalyzed hydrolysis of the penicilloyl enzyme with loss of peptidase activity gave rise to the defensive, penicillinhydrolyzing $\beta$-lactamases.

The monofunctional PBPs and the majority of the $\beta$-lactamases known today are acyl serine transferases. They fall into several amino acid sequence classes Similarity among members of a given class (i.e., intraclass similarity) forms a continuum, the cut-off points being $\approx 20 \%$ identities. Interclass similarity is almost nonexistent. Similarity is not always related to function. There is more similarity between the Streptomyces $\mathrm{K} 15$ DD-transpeptidase/PBP and the class A $\beta$-lactamases, or 


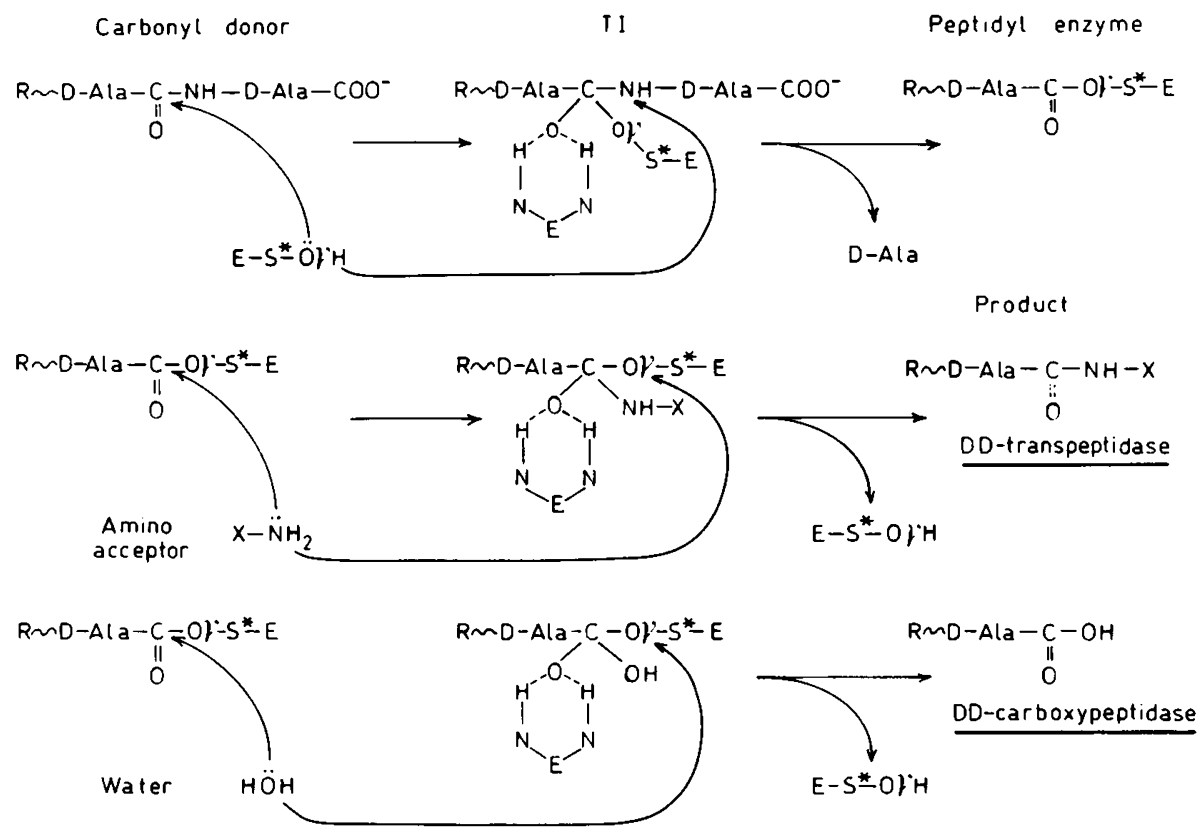

FIG. 3. Acyl transfer reactions on D-alanine-D-alanyl-terminated peptides via formation of a serine-ester-linked peptidyl enzyme. Attack of the peptidyl enzyme by an amino acceptor leads to transpeptidation of the carbonyl donor. Attack by water leads to hydrolysis. TI, terahedral intermediate.

between the Streptomyces R61 DD-carboxypeptidase/PBP and the class $\mathrm{C} \beta$-lactamases than between the class $\mathrm{A}$ and the class C $\beta$-lactamases.

The K15 PBP, the R61 PBP, and several class A and class $\mathrm{C} \beta$-actamases are of two domains (Fig. 5). ${ }^{18}$ One domain is of $\alpha$-helices and the other domain is a five-stranded $\beta$-sheet core that is covered by additional $\alpha$-helices. The active site that is sandwiched between the two domains, is a dense hydrogenbonding network interconnecting water molecules and the side
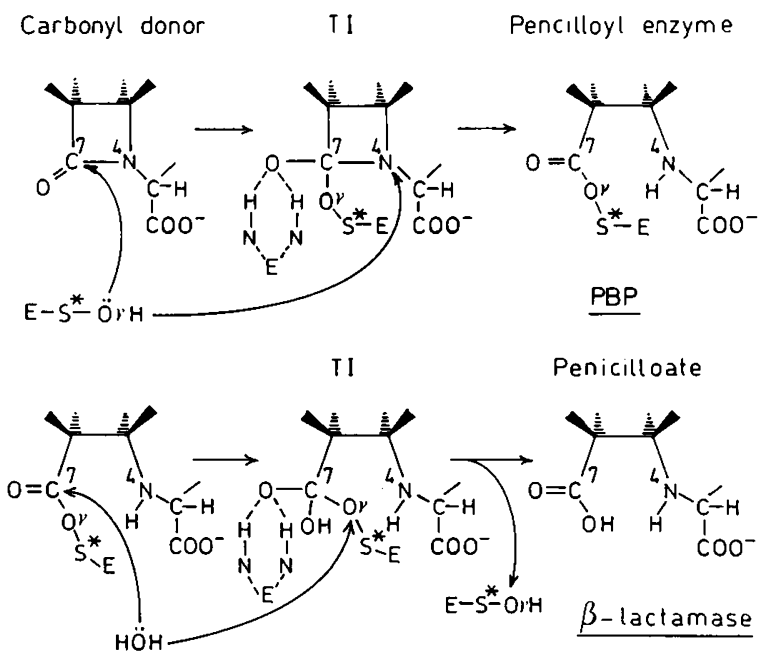

FIG. 4. Acyl transfer reactions on penicillin via formation of a serine-ester-linked penicilloyl enzyme. With the PBPs, the reaction stops, at least for a long time, at the level of the penicilloyl enzyme. With the $\beta$-lactamases, the reaction proceeds to hydrolysis of penicillin. chains of amino acid residues at the boundary of the cavity. This fold topology accommodates many local structural variations. With $\mathrm{x}$ denoting any amino acid residue, the tetrad $\mathrm{S}^{*} \mathrm{xxK}$ is located centrally at the amino end of an $\alpha$-helix of the all- $\alpha$ domain. The triad $[\mathrm{K} / \mathrm{H}][\mathrm{T} / \mathrm{S}] \mathrm{G}$ is on the innermost $\beta 3$ strand of the $\beta$-sheet on one side of the cavity, and the triad SGC (the $\mathrm{K} 15 \mathrm{PBP}$ ), YxN (the R61 PBP and the class $\mathrm{C} \beta$-lactamases), or SDN (the class A $\beta$-lactamases) is on a loop connecting two helices of the all- $\alpha$ domain on the other side of the cavity. The class $\mathrm{A} \beta$-lactamases have an additional active-site defining motif, the pentapeptide ExELN, located at the entrance of the cavity near the bottom of the $\beta-3$ strand. Compared with the K15 PBP and the class A $\beta$-lactamases, the R61 PBP and the class $C \beta$-lactamases have additional loops and secondary structures away from the active site.

In spite of differences in function, the monofunctional DDpeptidases/PBPs and $\beta$-lactamases have retained much of the same fold and much of the same active-site signature in the form of the motifs $S^{*} x x K,[S / Y] x N$ or analogue, and $[K / H / R][T / S] G$. They also have retained much of the same catalytic machinery. They each catalyze rupture of the $\beta$-lactam amide bond with transfer of the carbonyl carbon to the serine $\left(\mathrm{S}^{*}\right)$ residue and formation of a serine ester-linked penicilloyl enzyme. On the basis of this common property, they form a superfamily of peniciloyl serine transferases. They also catalyze acyl transfer on acyclic carbonyl donors

\section{R1-CONH-CH(R2)-COX-CH(R3)-COOH}

where $\mathrm{X}$ is $\mathrm{NH}, \mathrm{O}$, or $\mathrm{S}$. The substituents $\mathrm{R} 1, \mathrm{R} 2$, and $\mathrm{R} 3$, the nature of the scissile (peptide, ester, thiolester) bond, and the reaction pathways are class- and enzyme-specific., ${ }^{5,21}$

The specificity, fate, and rate of the catalyzed reactions de- 
pend on the accuracy of fit of the ligands (peptide, ester, thiolester, $\beta$-lactam carbonyl donors; amino acceptors) in the enzyme cavity. Catalysis also depends on the efficacy with which amino acid residues of the active site fulfill the required function of general base catalyst (abstracting the proton of the OHS $^{*}$ in step 1 and that of water or an amino acceptor in step 2) and provide an itinerary through which the abstracted proton can be back-donated to the right atoms in each step of the reaction.

An extensive study of the PBPs and $\beta$-lactamases of known three-dimensional structure by site-directed mutagenesis and molecular modeling has failed to identify, with certainty, the route that the proton uses during catalysis. ${ }^{17}$ At this level of the investigation, $10^{-10} \mathrm{~m}$, quantum effects rule the nanoworld and the proton shuttle can be disclosed only by the methods of quantum chemistry. Such methods are being developed. Studies carried out on chymotrypsin have led to the concept that the charge relay of an acyl serine transferase is created, de novo, by the interacting partners, the enzyme, and the ligand. 6.7 The creation of the charge relay results from the combined effects of the active-site environment, the deformation undergone by the bound ligand(s), the relaxation undergone by the enzyme polypeptide backbone, and the freedom of one or several water molecules. Hence, enzymes of a same family or even a same class can use more than one proton-shuttle route depending on structural features of the active site and/or the bound ligand. The naturally occurring $\beta$-lactamases of classes A, C, and D and the ease with which $\beta$-lactamase mutants emerge among clinical isolates support this concept. In recent years, at least $26 \beta$-lactamases of varying specificities have been identified. They each arose by alteration of amino acid residues in the class A TEM- $1 \beta$ lactamase. Evolution is occurring before our eyes.

In conclusion, the monofunctional penicilloyl serine transferases have evolved and are still evolving with preservation of a characteristic signature fold and much of the same serine-assisted acyl transfer machinery. They illustrate the principle according to which evolution obscures the function. The catalytic properties of a member of the superfamily cannot be deduced from its amino acid sequence and even fold topology. Direct biochemical evidence is required. Finally, the monofunctional penicilloyl serine transferases are highly adaptable structures. The essential serine residue may be activated by different general bases and several proton shuttle routes may be used.
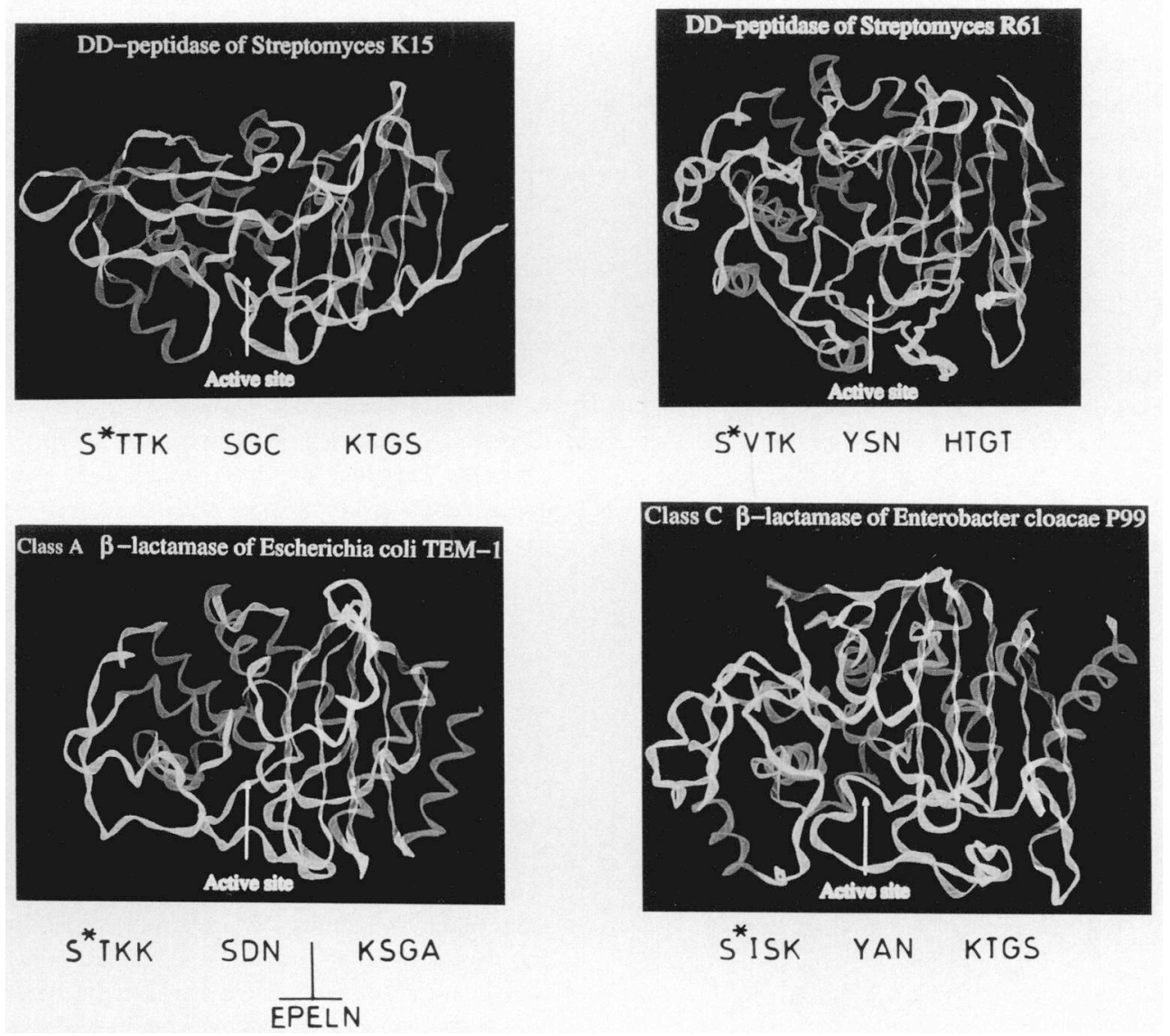

FIG. 5. Peptide fold of monofunctional penicilloyl serine transferases. The Streptomyces K15 DD-peptidase/PBP functions as a transpeptidase on D-alanyl-D-alanine-terminated peptides. The Streptomyces R61 DD-peptidase/PBP functions mainly as a carboxypeptidase. The E. coli TEM-1 $\beta$-lactamase and E. cloacae P99 $\beta$-lactamase are members of the amino acid sequence class $\mathrm{A}$ and class $\mathrm{C}$, respectively. The active-site defining motifs are indicated. For references, see Ghuysen. ${ }^{18}$ The atomic coordinates of the K15 PBP will be published shortly. 


\section{MULTIMODULAR WALL PEPTIDOGLYCAN HYDROLASES}

Monofunctional PBPs of $E$. coli are, at the same time, DD-carboxypeptidases and peptidoglycan hydrolases. They hydrolyze the carboxy terminal D-alanyl-D-alanine peptide bond (made by the D-Ala-D-Ala ligase) of the pentapeptide precursors. They also hydrolyze the carboxy terminal D-alanyl-(D)-meso-diaminopimelic acid bond (made by the transpeptidase) that cross-links the peptide unit in the completed peptidoglycan.

Streptomyces albus G secretes a non-penicillin-binding DDcarboxypeptidase/peptidoglycan hydrolase. This metallo (zinc) enzyme is constructed of two modules (Fig. 6). ${ }^{19}$ The arrow on the right points toward the zinc-containing active site borne by the 132 amino acid residue carboxy-terminal, catalytic (C) module. The arrow on the left points toward the cavity borne by the 81 amino acid residue amino-terminal, noncatalytic ( $\mathrm{n}-\mathrm{C})$ module. The crevice (18.6 $\AA / 13.5 \AA$ ) of the $n-C$ module is defined by two $\alpha$-helical repeats ( $\alpha 2$ and $\alpha 3$ ) each 16 amino acid residues long, connected by a heptapeptide loop.

The $\mathrm{n}-\mathrm{C}$ module of the $\mathrm{Zn}$ DD-peptidase is the prototype of an amino acid sequence family of $\mathrm{n}-\mathrm{C}$ modules also found in the Bacillus subtilis CwlA and $B$. licheniformis CwlL $N$-acetylmuramoyl-L-alanine amidases and the Corynebacterium acetobutylicum $\mathrm{N}$-acetylmuramidase (lysozyme). Similarity between the $\mathrm{n}-\mathrm{C}$ modules is high (30-50\% identities), and, therefore, one can be confident that they have the same fold. However (with IP denoting an intervening peptide) the modular design of the peptidoglycan hydrolases is different:

$\mathrm{NH}_{2}-[\mathrm{n}-\mathrm{C}]-[\mathrm{C}]-\mathrm{COOH}$ for the $\mathrm{Zn}$ DD-peptidase

$\mathrm{NH}_{2}-[\mathrm{C}]-[\mathrm{n}-\mathrm{C}]-\mathrm{COOH}$ for the $\mathrm{CwlA}$ amidase

$\mathrm{NH}_{2}-[\mathrm{C}]-[\mathrm{n}-\mathrm{C}]-[\mathrm{n}-\mathrm{C}]-\mathrm{COOH}$ for the lysozyme

$\mathrm{NH}_{2}-[\mathrm{C}]-[\mathrm{n}-\mathrm{C}]-\mathrm{IP}-[\mathrm{n}-\mathrm{C}]-\mathrm{COOH}$ for the CwlL amidase

Depending on the enzymes, the $\mathrm{n}-\mathrm{C}$ module occurs at the amino or the carboxy end of the $\mathrm{C}$ module in one or two copies and the copies are either contiguous or connected by an intervening peptide.

Acquisition of a substrate-binding module fused to the catalytic module is an evolutionary advantage for exocellular enzymes that interact with and hydrolyze bonds in a polymeric substrate. Peptidoglycan hydrolases have achieved this feat

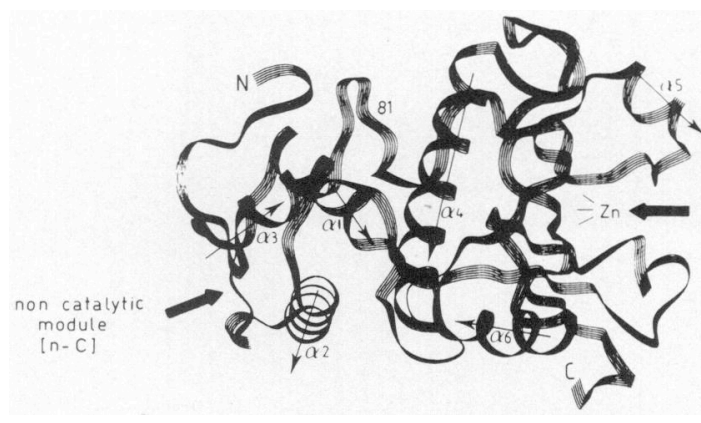

FIG. 6. Peptide fold of the bimodular Zn DD-carboxypeptidase/endopeptidase of Streptomyces albus $G$. Reproduced from Ghuysen et al., ${ }^{19}$ with permission of Elsevier. through the interchange and local structural alterations of specialized modules. The likely function of these modules as substrate recognition/binding sites would depend mainly on the conserved amino acid residues. Substrate specificity and directed topological activity would depend on the occurrence of nonconserved amino acid residues and the location and copy number of the $\mathrm{n}-\mathrm{C}$ modules.

\section{BIMODULAR PENICILLIN RECEPTORS}

The bacterial cells are sensitive to virtually every aspect of their environment and these environmental changes are monitored by specialized sensory transducers. The dominant forms of signal transduction proceed via phosphoryl transfer pathways (the so-called two-component regulatory systems) or are associated with sites of methylation and demethylation.

Bacteria have developed unique transduction pathways that detect $\beta$-lactam molecules in the environment of the cell and switch on the transcription of the $\beta$-lactamase encoding gene. Induction of $\beta$-lactamase synthesis in a number of gram-negative bacteria is the result of $\beta$-lactam antibiotic-induced and peptidoglycan hydrolases-mediated deregulation of the cell wall recycling process. ${ }^{26,27}$ In the gram-positive Bacillus licheniformis, the induction of $\beta$-lactamase synthesis rests upon the presence in the membrane, of a penicillin receptor that results from a fusion event through which a penicilloyl serine transferase polypeptide is linked to the carboxy end of a signal transducer. ${ }^{29}$

Regulation of transcription of the $\beta$-lactamase-encoding blaP gene in $B$. licheniformis involves three-chromosome-borne regulatory genes. blal encodes a $15-\mathrm{kDa}$ repressor; blaRl encodes a penicillin receptor; the product of blaR 2 is of unknown function. Membrane topology experiments, predictional studies, and conformational analyse ${ }^{29}$ (unpublished data from this laboratory and R. Brasseur) strongly suggest that the 601 amino acid residue blaRI-encoded penicillin receptor BlaR has the multipartite organization shown in Figure 7. Central to this model is a four $\alpha$-helix bundle defined by four transmembrane segments TM1 to TM4. A 63 amino acid residue extracellular domain connects TM2 and TM3. A 189 amino acid residue intracellular domain that possesses the signature of a metallo-peptidase $\left(\mathrm{Zn}^{2+}\right.$ binding site) connects TM3 and TM4. A 261 amino acid residue extracellular domain, the penicillin sensor, is fused to the carboxy end of TM4. This sensor possesses the active-site defining motifs ( ${ }^{*}$ TYK, YCN, KTGT) of the penicilloyl serine transferases superfamily. The sensor can be produced independently from the rest of BlaR as a water-soluble polypeptide in the periplasm of $E$. coli. The isolated polypeptide binds penicillin and behaves as a high affinity PBP.

On the basis of this model, a likely and testable mechanism of signal transmission by BlaR may be put forward. Penicillininduced conformational changes in the penicillin-bound sensor and the interacting 63 amino acid residue extracellular domain would be transmitted via the four $\alpha$-helix bundle to the intracellular domain with concomitant activation of the putative metallopeptidase. Degradation of the BlaI repressor or release of an antirepressor in the cytosol by the "activated" peptidase would result in derepression of $\beta$-lactamase synthesis.

BlaR is the prototype of an amino acid sequence family of penicillin receptors also involved in the penicillin-induced $\beta$-lactamase 


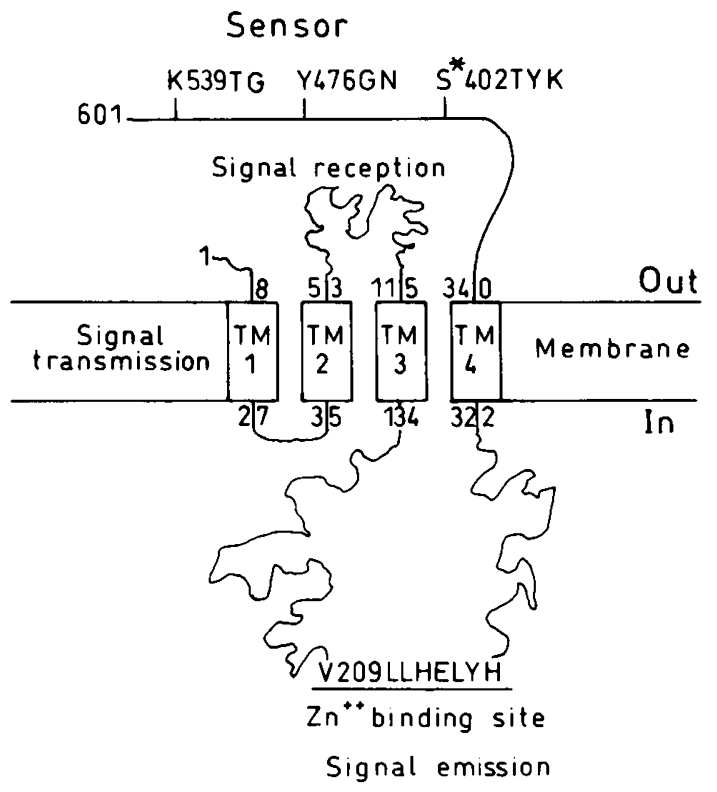

FIG. 7. Schematic representation of the bimodular penicillinreceptor BlaR of $B$. licheniformis. The penicillin sensor belongs to the amino acid sequence class $D \beta$-lactamases.

and low-affinity PBP2' synthesis in S. aureus. ${ }^{29}$ The S342-R601 polypeptide sensor of BlaR is, itself, a member of the amino acid sequence class D $\beta$-lactamases ( $32 \%$ identities with the Oxa- $2 \beta$ lactamase), suggesting a common signature fold. Hence, a penicilloyl serine transferase of a given class (a $\beta$-lactamase) may acquire a new property (penicillin sensing) through local changes and fusion to another polypeptide, and the resulting chimeric protein may acquire a new function (gene regulation).

\section{MULTIMODULAR PENICILLIN-BINDING PROTEINS}

The high-molecular-mass PBPs are also of modular design ${ }^{12}$ (Table 1, Fig. 8). The amino acid sequence data are from the literature. 2,12,34

A penicillin-binding ( $\mathrm{PB}$ ) module that belongs to and possesses the $S^{*} \times x K, S x N$, and $K[T / S] G$ markers of the penicill- loyl serine transferases superfamily is fused to the carboxy end of a non-penicillin-binding (n-PB) module in a single polypeptide chain that folds on the outer face of the plasma membrane. The polypeptide itself is fused to an amino-terminal transmembrane-anchoring module. Inserts may occur that are large enough to form additional modules. By analogy with the monofunctional PBPs, the PB modules are assumed to start about 60 amino acid residues upstream from the $S^{*} \times x K$ motif and to terminate about 60 amino acid residues downstream from the $\mathrm{K}[\mathrm{T} / \mathrm{S}] \mathrm{G}$ motif. The $\mathrm{n}-\mathrm{PB}$ modules also possess specific amino acid markers, but the signature of the $n-P B$ modules of the class A multimodular PBPs is different from that of the n-PB modules of the class B multimodular PBPs.

As a polypeptide chain increases in length, finding the right fold becomes more difficult because the possibilities of misfolding increase. Study of derivatives of multimodular PBPS overproduced from appropriate expression (and secretion) vectors shows that the acquisition of a stable, penicillin-binding fold topology by the PB module is membrane-anchor independent but requires concomitant biogenesis of the n-PB module. As shown with the E. coli PBP1b ${ }^{31}$ and PBP3 ${ }^{16}$ (unpublished data), the $S$. aureus PBP2',40,41 and the E. hirae PBP5 (unpublished data), replacement of the membrane anchor by a cleavable signal peptide or substitution of the genuine anchor by another transmembrane anchoring device has no effect on the thermostability and penicillin-binding capacity of the PBP mutants. However, elimination of both the membrane anchor and the n-PB module (or part of it) or elimination of the n-PB module with conservation of the membrane anchor is not tolerated. Moreover, expression of $E$. coli ftsl genes encoding PBP3 mutants in which E193 of motif $3^{*}$ of the n-PB module is replaced by $\mathrm{D}$ or $\mathrm{N}$ gives rise to membrane-bound proteins that are very unstable, suggesting that motif $3^{*}$ plays a role in the folding process (unpublished data from this laboratory and J. Ayala).

Intraclass similarity between the n-PB modules and the PB modules, respectively, is a continuum with a cut-off point of about $20 \%$ identities (Table 2). Interclass similarity between the $\mathrm{n}-\mathrm{PB}$ modules is nonexistent and interclass similarity between the PB modules is very low. The PB modules of the class A PBPs and those of class B PBPs have diverged so far that traces of similarity other than the active site defining motifs have almost completely disappeared. The n-PB modules of the class A PBPs and those of the class B PBPs form two distinct fam-

Table 1. Size of the Multimodular PbPs in Number of Amino Acid Residues and Position of the Active Site Serine S*

\begin{tabular}{|c|c|c|c|c|c|}
\hline Class A & $\alpha \alpha$ & $S^{*}$ & Class B & $\alpha \alpha$ & $S^{*}$ \\
\hline 1. B. subtilis $1 \mathrm{a} / \mathrm{b}$ & 914 & 390 & 1. S. pneumoniae $2 \mathrm{x}$ & 750 & 337 \\
\hline 2. H. influenzae $1 \mathrm{a}$ & 864 & 452 & 2. S. pneumoniae $2 \mathrm{~b}$ & 680 & 385 \\
\hline 3. E. coli $1 \mathrm{a}$ & 850 & 465 & 3. E. hirae $5,3^{r}$ & 678 & 422 \\
\hline 4. E. coli $\mathrm{lb}$ & 844 & 510 & 4. S. aureus 2' & 667 & 405 \\
\hline 5. M. leprae 1 & 821 & 398 & 5. E. coli 2 & 633 & 330 \\
\hline 6. S. aureus $2 \mathrm{x}$ & 727 & 398 & 6. E. coli 3 & 588 & 307 \\
\hline 7. S. pneumoniae la & 719 & 370 & 7. N. gonorrhoeae 2 & 582 & 312 \\
\hline 8. B. subtilis la & 714 & 359 & 8. N. meningitidis & 582 & 310 \\
\hline 9. $S$. oralis 1a & 637 & 371 & & & \\
\hline 10. B. subtilis 4 & 624 & 388 & & & \\
\hline
\end{tabular}




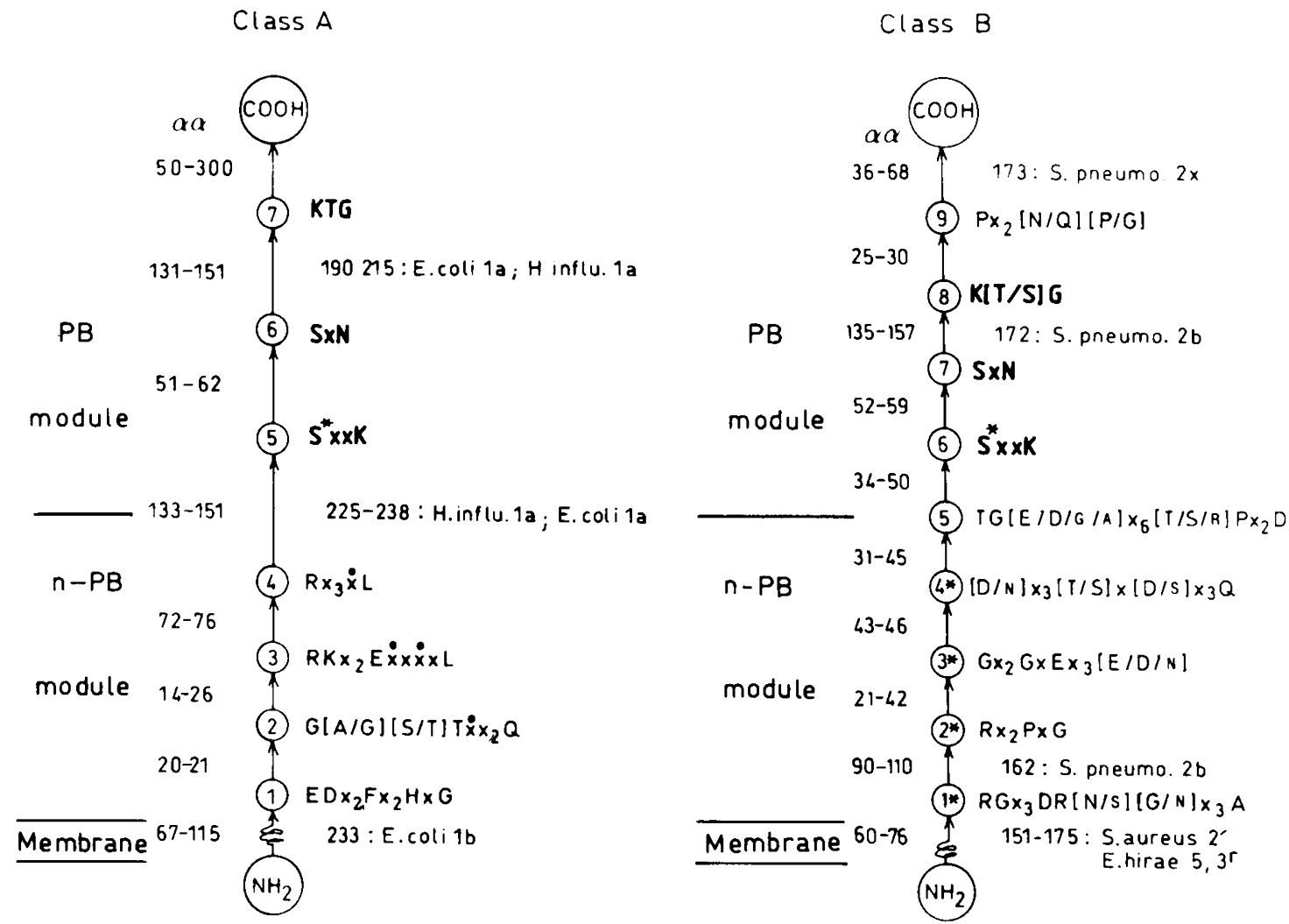

FIG. 8. Design and amino acid sequence signatures of the multimodular PBPs of classes A and B. The distribution of the conserved motifs, the average length of the intermotif sequence, and the occurrence of inserts (expressed in number of amino acid residues $\alpha \alpha$ ) are shown. The data derive from the amino acid sequences of the PBPs listed in Table 1. S*, active-site serine; $\mathrm{x}$, variable amino acid residue; $\dot{x}$, hydrophobic amino acid residue.

Table 2. Identities (\%) Between the Aligned Amino Acid Sequences of High-Molecular-Mass PBPs ${ }^{a}$

\begin{tabular}{|c|c|c|c|c|c|c|c|c|c|c|c|c|c|c|c|c|c|c|}
\hline \multirow[b]{2}{*}{$P B P$} & \multicolumn{9}{|c|}{$n-P B$ module } & \multicolumn{9}{|c|}{ PB module } \\
\hline & 1 & 2 & 3 & 4 & 5 & 6 & 7 & 8 & 9 & 1 & 2 & 3 & 4 & 5 & 6 & 7 & 8 & 9 \\
\hline & \multicolumn{4}{|c|}{ Class A } & \multicolumn{5}{|c|}{ Interclass } & \multicolumn{4}{|c|}{ Class A } & \multicolumn{5}{|c|}{ Interclass } \\
\hline \multicolumn{19}{|l|}{ Class A } \\
\hline 1. M. leprae & 100 & 26 & 27 & 24 & 12 & 7 & 8 & 6 & 6 & 100 & 17 & 16 & 18 & 10 & 11 & 12 & 11 & 10 \\
\hline 2. E. coli 1a & & 100 & 57 & 22 & 8 & 6 & 8 & 7 & 7 & & 100 & 52 & 24 & 10 & 11 & 12 & 12 & 11 \\
\hline $\begin{array}{l}\text { 3. H. influ- } \\
\text { enzae la }\end{array}$ & & & 100 & 24 & 9 & 8 & 7 & 5 & 7 & & & 100 & 24 & 11 & 14 & 13 & 13 & 11 \\
\hline 4. E. coli $1 \mathrm{~b}$ & & & & 100 & 9 & 6 & 8 & 7 & 8 & & & & 100 & 10 & 12 & 10 & 10 & 10 \\
\hline Class B & \multicolumn{9}{|c|}{ Class B } & & & & & \multicolumn{5}{|c|}{ Class B } \\
\hline 5. E. coli 2 & & & & & 100 & 26 & 22 & 20 & 20 & & & & & 100 & 24 & 28 & 17 & 23 \\
\hline 6. E. coli 3 & & & & & & 100 & 19 & 17 & 19 & & & & & & 100 & 21 & 20 & 17 \\
\hline 7. E. hirae $3^{r}$ & & & & & & & 100 & 72 & 28 & & & & & & & 100 & 85 & 39 \\
\hline 8. E. hirae 5 & & & & & & & & 100 & 28 & & & & & & & & 100 & 40 \\
\hline $\begin{array}{l}\text { 9. S. aureus } \\
2^{\prime}\end{array}$ & & & & & & & & & 100 & & & & & & & & & 100 \\
\hline
\end{tabular}

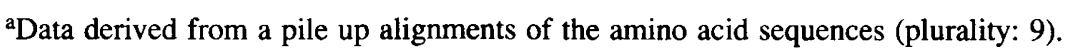


ilies. The two families have a characteristic amino acid sequence and, presumably, fold signature, suggesting that they have evolved from different polypeptide ancestors. As an n-PB module of class $A$ is linked to a PB module of class $A$ and an n-PB module of class $B$ is linked to a PB module of class $B, a$ likely corollary of this class-specific modular design is that the class A PBPs perform a different function (or different functions) from that (those) of the class B PBPs. In consequence, the effects of the inactivation of the class A PBPs on the cell viability should be different from those of the class B PBPs.

In vitro, the purified class A PBP1a and PBP1b of E. coli (and, perhaps, by extension other class A PBPs) are wall peptidoglycan synthetases. They catalyze glycan chain elongation and peptide cross-linking from the lipid II intermediate. Inhibition of the n-PB/transglycosylase module of PBP1b by moenomycine prevents peptide cross-linking while inactivation of the $\mathrm{PB} /$ transpeptidase module by penicillin enhances glycan chain elongation, showing that the two modules interact with each other. Moreover, PBP1b contains a membrane association site in addition to the transmembrane anchor ${ }^{31}$ and dimeric forms of PBP1b are in close association with the peptidoglycan. ${ }^{42}$

In vivo, inactivation of the $\mathrm{PB}$ modules of PBP1a and PBP1b of $E$. coli by $\beta$-lactam antibiotics causes peptidoglycan hydrolases-induced cell lysis. Deletion of the genes encoding PBP1a and PBP1b is fatal, but deletion of either of the PBP1a- or the PBP1b-encoding gene is tolerated, suggesting that one PBP can compensate for another.

In vitro, the purified class B PBP3 of E. coli ${ }^{21}$ (unpublished data) and PBP2x of $S$. pneumoniae ${ }^{28}$ catalyze serine-assisted hydrolysis and aminolysis of thiolester carbonyl donors. Bacterial strains having a reduced affinity for penicillin and possessing one or several class B PBPs with reduced affinity for the drug synthesize a wall peptidoglycan with a different peptide moiety from that of the wild type. ${ }^{36} \mathrm{Hence}$, the PB modules of the class B PBPs are involved, one way or another, in peptidoglycan cross-linking. However, the precise nature of the catalyzed reactions remains to be elucidated. Indeed, the isolated PBP3 of $E$. coli is inert on the lipid II intermediate ${ }^{1}$ (unpublished results from this laboratory and J. Van Heijenoort). Moreover, evolution may obscure the function of a protein and fusion between polypeptides may result in the acquisition of a new function (see preceding sections).

Given that the n-PB modules of the class B PBPs have a different amino acid signature from that of the $\mathrm{n}$-PB modules of the class A PBPs, they may not have a transglycosylase activity. The acyl transferase activity of their PB modules might be coupled with the transglycosylase activity of the $\mathrm{n}$-PB modules of some class A PBPs. Such a situation implies that the class $A$ and class B multimodular PBPs interact with each other. One may also note that the $E$. coli $\mathrm{PBP} 3$ forms dimers (personal communication from N. Nanninga and J. Ayala).

In vivo, the primary effects of the inactivation of the $\mathrm{PB}$ modules of the class B PBP2 and PBP3 of $E$. coli by $\beta$-lactam antibiotics are morphological abnormalities, followed by cell lysis. Inactivation of PBP2 results in growth as spherical cells (the rod-shaped maintenance machinery is no longer functional). Inactivation of PBP3 results in growth as filamentous cells (the septation machinery is no longer functional). Inactivation of either of the PBP2- or PBP3-encoding genes of
E. coli and of the PBP2b- or PBP2x-encoding genes of S. pneumoniae is not tolerated.

Like the monofunctional PBPs and $\beta$-lactamases, the multimodular PBPs are highly adaptable structures. Multimodular PBP-mediated resistance to $\beta$-lactam antibiotics among grampositive pathogens has become a serious health problem.

In S. pneumoniae, ${ }^{10,23,38}$ the first PBPs to be affected during selection of laboratory mutants having a reduced affinity for cefotaxime and piperacillin are the class B PBP2x and PBP2b, respectively. The modified PBP2x and PBP2b confer resistance upon transformation. Low affinity forms of PBP2x and PBP2b and the class A PBP1a are present in highly resistant clinical isolates. Reduced affinity is the result of structural changes affecting either a limited number of amino acid residues or large blocks of amino acid sequences of the PB modules. Interspecies recombinational events occur that replace part of a PBP-encoding gene with the corresponding parts from homologous PBP-encoding genes of closely related, naturally resistant species.

The low-affinity class B PBP2' of S. aureus ${ }^{3}$ and PBP5 and $\mathrm{PBP} 3 \mathrm{r}$ of $E$. hirae $e^{11,33}$ allow the strains that produce them to grow and manufacture a wall peptidoglycan under conditions in which all the monofunctional PBPs and the PB modules of all the other multimodular PBPs are inactivated by penicillin. The staphylococcal PBP2' is chromosomal and, in some strains, its penicillin-induced synthesis is BlaR-mediated. ${ }^{29}$ The enterococcal PBP5 is also chromosomal but its homologue PBP3r is plasmid borne in $E$. hirae S185R (unpublished data). This plasmid carries, at least, two copies of the PBP3r-encoding gene, one copy of the streptomycin-resistance marker $s t r$, one copy of the erythromycin-resistance marker $\mathrm{erm}$, and several copies of the insertion module IS 1216 , a situation eminently favorable for the spread of multiresistance among enterococci and related bacterial species.

As the origin of the low-affinity class B PBPz, PBPs, and PBP3r is unknown, the structural features responsible for the low affinity are also unknown. Compared to the other class B multimodular PBPs, the low-affinity PBPs have an extended $\mathrm{n}$-PB module because of the presence of $\mathrm{a} \approx 100$ amino acid residue insert immediately downstream from the membrane anchor. These inserts are related in amino acid sequence. Presumably, they have a same fold. Expression of genes encoding low-affinity PBP derivatives in which the insert is missing or truncated gives rise to proteins that are inert in terms of penicillin-binding ${ }^{41}$ (unpublished data), showing that the insert plays a role in biogenesis. The active-site topology of the PB module confers resistance to the usual penicillins and cephalosporins but not necessarily to all $\beta$-lactam compounds. Cephalosporin derivatives are being developed that are active against methicillin-resistant $S$. aureus strains. ${ }^{22}$

A plausible picture that arises from the above analysis is that the enzyme activities required to build the peptidoglycan poly-

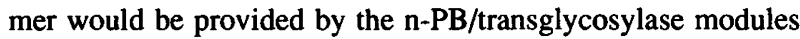
of the class A PBPs and the PB/acyl transferase modules of both class A and class B PBPs. Regulation of these activities in a cell-cycle-dependent fashion would be mediated by the $\mathrm{n}$ PB modules of the class B PBPs. Recent genetic studies have brought to light the existence of morphogenetic networks. These networks are multiprotein complexes, the constitutive elements 
of which are sets of multimodular PBPs, monofunctional PBPs, and non-penicillin-binding proteins.

\section{MORPHOGENETIC NETWORKS}

Figure 9 shows part of the gene organization in the chromosome of E. coli. The 4 and $75 \mathrm{~min}$ regions contain the genes encoding the bienzyme (transglycosylase/transpeptidase) class A PBP1b and PBP1a, respectively.

The 2 min region, which contains the gene encoding the multimodular class B PBP3, also contains genes that encode other cell-septation proteins. ${ }^{1,9}$ Figure 10 shows the cellular localization of some components of the cell septation network (also called divisome or septator). FtsQ is of unknown function and FtsL contains a leucine zipper motif. Each is on the outer face of the plasma membrane. FtsW is an integral membrane protein. FtsA, an ATP-binding protein of the eukaryotic actin family, ${ }^{35}$ is exposed on the inner face of the plasma membrane. MraW is a protein bearing an $S$-adenosyl methionine-binding motif and FtsZ is a GTP-binding/GTPase protein. Each is cytoplasmic. FtsZ contains a short glycine-rich segment that is strikingly similar to the GTP-binding motif of the eukaryotic cytoskeletal tubulin. ${ }^{13}$ About 10,000 molecule of FtsZ occur per cell. They form a ring at the future division site ${ }^{39}$ in the form of $\mathrm{a} \approx 40-\mu \mathrm{m}$ filament that is long enough to surround the

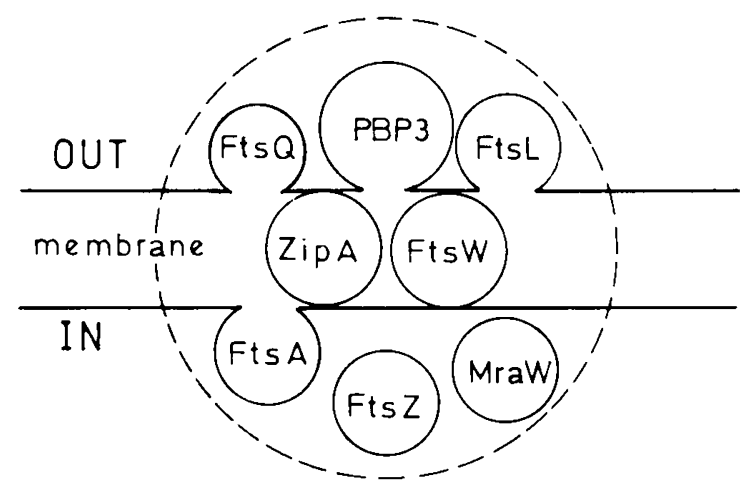

FIG. 10. Cellular localization of proteins of the "cell septation" morphogenetic network in E. coli.

cell 20 times. These genes form a cluster the expression of which is controlled by a gearbox and metabolic promoters.

Genes located outside the $2 \mathrm{~min}$ region of the chromosome also encode "cell septation" proteins. SulA is a cell-division inhibitor. ZipA is another integral membrane protein. Slt-70 is an $\mathrm{N}$-acetylmuramidase that catalyzes the hydrolysis of the glycosidic bond with transfer of the carbonyl to the C-6 hydroxyl group, yielding a (1-6)anhydromuramic acid. ${ }^{25}$ FtsK possesses probably an $\mathrm{N}$-terminal domain with several membrane-spanning helices and a large cytoplasmic domain with an ATP/GTP-

\section{Lipid II intermediate synthesis 7}
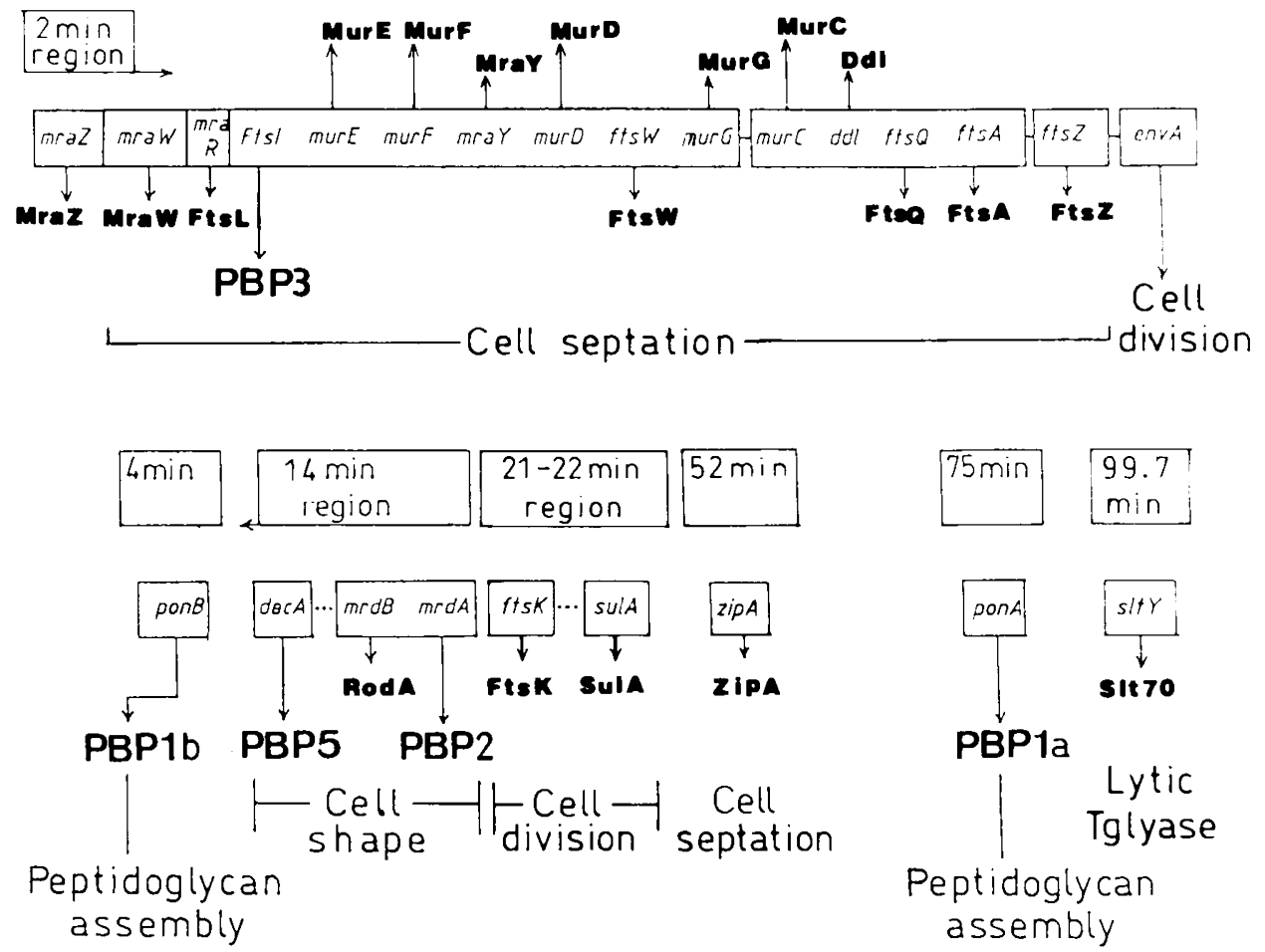

FIG. 9. Organization of the genes in the chromosome of $E$. Coli involved in lipid II intermediate synthesis, peptidoglycan assembly, cell septation, cell shape, and cell division. ponA and ponB are also called $\operatorname{mrcA}$ and $\operatorname{mrcB}$, respectively. Tglyase, transglycosylase. 
binding motif (L. Begg, S. Dewar, and W. Donachie; report presented at the workshop "Structure, Function and Controls in Microbial Division." Institute Juan March, Madrid, May 1995). A single base change in $f t s \mathrm{~K}$ causes a conditional block in cell division that is suppressed by deletion of the PBP5-encoding dacA.

A battery of techniques is being used to study protein-protein interactions within the "cell septation network." According to reports presented at the workshop mentioned above, PBP3 interacts, presumably through its intracellular amino end, with FtsA, SulA, and FtsZ in the cytoplam; FtsZ itself interacts with the integral membrane protein ZipA; and PBP3 interacts, presumably through its periplasmic module, with PBP1b, PBP7 (an endopeptidase), and Slt-70.

At the same time, the structural requirements of PBP3 for in vivo activity are being studied by complementation experiments. In $E$. coli $\mathrm{RP} 41$, the temperature-sensitive-ftsI 2158-encoded PBP3 has two mutations: G191-D in motif $3^{*}$ of the n$\mathrm{PB}$ module and D266-N at the junction between the n-PB and $\mathrm{PB}$ modules. ${ }^{1}$ At $42^{\circ} \mathrm{C}$, E. coli $\mathrm{RP} 41$ grows as filaments and lyses but rod-shaped, cell division and viability are restored by transformation with a plasmid carrying the wild-type ftsI. In contrast, complementation is not achieved by $f t s I$ genes encoding PBP3 mutants that either lack the membrane anchor, have a membrane anchor different form the gènuine one, or have a 17 amino acid residue insert immediately upstream from R60 of motif $1^{*}$ (the insert being the R60-D76 sequence, except that D76 is mutated into N; unpublished data from this laboratory and $\mathrm{J}$. Ayala). Complementation does not occur in spite of the fact that the produced PBP3 mutants have the same thermostabiltiy and penicillin-binding capacity as the wild-type PBP3.

The acquisition of a penicillin-binding fold topology by the PB module of PBP3 depends on an intact motif $3 *$ of the n-PB module but it is membrane-anchor-independent. It now appears that the in vivo activity of PBP3 requires not only a correct penicillin-binding fold topology, but, in addition, the presence of the genuine membrane anchor and an intact environment of motif $1 *$ of the $n-P B$ module. It has also been reported that the P565-G571 sequence at the end (V577) of the mature PBP3 is not required for penicillin-binding but is essential for in vivo activity. ${ }^{20}$ Likely, the membrane anchor, features of the n-PB module, and the carboxy end of the polypeptide chain are sites through which PBP3 may interact with other components of the "cell division" morphogenetic network.

The $14 \mathrm{~min}$ region of the $E$. coli chromosome, which contains the gene encoding the multimodular class B PBP2, also contains genes that encode the "cell-shape" PBP5 and RodA, a protein very similar to FtsW. This complex and ribosomal activities appear to be coordinated by a chain of interacting elements, one of which is regulated by the nucleotide guanosine 5'-diphosphate, 3'-diphosphate (ppGpp, an RNA polymerase effector), itself synthesized by the SpoT/RelA, proteins. Remarkably, the "cell shape" and "cell septation" morphogenetic networks are connected. PBP2 is not required for septum synthesis. However, loss of PBP2 activity results in a block of cell division and, in the absence of PBP2, cell division and viability are restored by increasing the pool of ppGpp or the level of FtsQ-A-Z. ${ }^{30}$

The "cell septation" and "cell shape" networks are probably ubiquitous in the bacterial world, with many individual varia- tions. Likely, other networks remain to be identified. The stepwise increased resistance to $\beta$-lactam compounds of $S$. pneumoniae laboratory mutants does not always correlate with PBP changes, but correlates with genetic competence deficiency. ${ }^{23}$ In a cefotaxime laboratory mutant, increased resistance and competence deficiency are mediated by a point mutation in a histidine kinase $\mathrm{CiaH} .{ }^{23} \mathrm{CiaH}$ and $\mathrm{CiaR}$ are members of signal transduction pathways including the EnvZ/OmpR osmoregulation in E. coli and the VanS/VanR vancomycin resistance inducibility in E. faecium.

The morphogenetic networks are still far from being understood. They probably possess several phosphoryl transfer pathways. They may be components of multiple parallel, overlapping, and interacting signal transduction systems. There is cross-talk between the pathways. These characteristics are typical of "(phospho?)neural" networks. ${ }^{24}$

\section{CONCLUSION}

The synthesis and assembly of the bacterial cell wall peptidoglycan require proteins the primary functions of which are the chemical transformation of metabolite intermediates, the building of a cellular structure, and the transfer and processing of information through integrated biochemical "circuits" that are able to transform an input signal into an output signal and an output signal into an input signal.

The wall peptidoglycan is a bacterium-specific polymer. Empirical approaches to the discovery of "bacterial-cell-wall" inhibitors and the improvement of existing drugs were justified when basic research was still struggling to cope with the complexity of the process and the molecular structures. The situation is changing. With the present advanced state of our knowledge and the availability of experimental and theoretical tools of ever increasing incisiveness, future antibacterial chemotherapy strategies are likely to depend on the understanding of the functioning of existing targets at the atomic level allowing new drugs to be designed, and on the identification of the constitutive elements and the "wiring" of the morphogenetic networks allowing new targets to be discovered.

\section{ACKNOWLEDGMENTS}

This work was supported in part by the Belgian program on Interuniversity Poles of Attraction initiated by the Belgian State, Prime Minister's Office, Services fédéraux des affaires scientifiques, techniques et culturelles (PAI 19), the Fonds de la Recherche Scientifique Médicale (contract 3.4531.92), and the Fonds de la Recherche Fondamentale Collective (contract 2.4534.95)

\section{REFERENCES}

1. Ayala, J.A., T. Garrido, M.A. de Pedro, and M. Vicente. 1994. Molecular biology of bacterial septation, pp. 73-101. In R. Hakenbeck and J.M. Ghuysen (eds.), New comprehensive biochemistry, Vol. 27, Bacterial cell wall. Elsevier Science Publishers, Amsterdam. 
2. Basu, J., S. Mehapatra, M. Kundu, S. Mukhopadhyay, M. Nguyen-Distèche, P. Dubois, B. Joris, J. Van Beeumen, P. Chakrabarti, S.T. Cole, and J.M. Ghuysen. 1996. Identification and overexpression in Escherichia coli of a Mycobacterium leprae gene, pon 1, encoding a high-molecular-mass class A penicillinbinding protein PBP1. J. Bacteriol. 178:1707-1711.

3. Berger-Bächi, B. 1994. Expression of resistance to methicillin. Trends Microbiol. 2:389-393.

4. Brown, E.D., E.I. Vivas, C.T. Walsh, and R. Kolter. 1995. MurA (MurZ), the enzyme that catalyzes the first committed step in peptidoglycan biosynthesis, is essential in Escherichia coli J. Bacteriol. 177:4194-4197.

5. Damblon, C., G.H. Zhao, M. Jamin, P. Ledent, A. Dubus, M. Vanhove, X. Raquet, L. Christiaens, and J. M. Frère. 1995. Breakdown of the stereospecificity of DD-peptidases and $\beta$-lactamases with thiolester substrates. Biochem. J. 309:431-436.

6. Dive, G., D. Dehareng, and J.M. Ghuysen. 1994. A detailed study of a molecule into a molecule: The $\mathrm{N}$-acetyl-L-tryptophanamide in active site model of the d-chymotrypsin. J. Am. Chem. Soc. 116:2548-2556.

7. Dive, G., D. Dehareng, and D. Peeters. 1996. Proposition for the acylation mechanism of the serine proteases: A one-step process? Int. J. Quant. Chem. 58:85-107.

8. Dobson, C.M. 1995. Finding the right fold. Struct. Biol. 2:513-517.

9. Donachie, W.D. 1992. Regulation of the morphogenetic cycle of Escherichia coli, pp. 409-419. In M.A. de Pedro, J.V. Höltje, and W. Löffelhardt (eds.), Bacterial growth and Lysis: Metabolism and structure of the bacterial sacculus. Plenum Press, New York.

10. Dowson, C.G., T.J. Coffey, and B.G. Spratt. 1994. Origin and molecular epidemiology of penicillin-binding-protein-mediated resistance to $\beta$-lactam antibiotics. Trends Microbiol. 3:361-366.

11. El Kharroubi, A., P. Jacques, G. Piras, J. Coyette, J. Van Beeumen, and J.M. Ghuysen. 1991. The Enterococcus hirae R40 penicillin-binding protein 5 and the methicillin-resistant Staphylococcus aureus penicillin-binding protein $2^{\prime}$ are homologs. Biochem. J. 280:463-469.

12. Englebert, S., G. Piras, A. El Kharroubi, B. Joris, J. Coyette, M. Nguyen-Distèche, and J.M. Ghuysen. 1993. Modular design of the bi(multi?)functional penicillin-binding proteins, pp. 319-333. In M.A. de Pedro, J.V. Höltje, and W. Löffelhardt (eds.), Bacterial growth and lysis: Metabolism and structure of the bacterial sacculus. Plenum Press, New York.

13. Erickson, H.P. 1995. FtsZ, a prokaryotic homolog of tubulin? Cell 80:367-370.

14. Fan, C., P.C. Moews, C.T. Walsh, and J.R. Knox. 1994. Vancomycin resistance: Structure of D-alanine:D-alanine ligase at $2.3 \AA$ resolution. Science 266:439-443.

15. Fan, C., P.C. Moews, C.T. Walsh, and J.R. Knox. 1995. A common fold for peptide synthetases cleaving ATP to ADP: Glutathione synthetase and D-alanine:D-alanine ligase of Escherichia coli. Proc. Natl. Acad. Sci. U.S.A. 92:1172-1176.

16. Fraipont, C., M. Adam, M. Nguyen-Distèche, W. Keck, J. Van Beeumen, J.A. Ayala, B. Granier, H. Hara, and J.M. Ghuysen. 1994. Engineering and overexpression of periplasmic forms of the penicillin-binding protein 3 of Escherichia coli. Biochem. J. 298: 189-195.

17. Frère, J.M. 1995. $\beta$-Lactamases and bacterial resistance to antibiotics. Mol. Microbiol. 16:385-395.

18. Ghuysen, J.M. 1994. Molecular structures of penicillin-binding proteins and $\beta$-lactamases. Trends Microbiol. 2:372-380.

19. Ghuysen, J.M., J. Lamotte-Brasseur, B. Joris, and G.D. Shockman. 1994. Binding site-shaped repeated sequences of Streptomyces albus G DD-peptidase, Bacillus subtilis CwlA amidase, Corynebacterium acetobutylicum lysozyme and Bacillus sp ORF-L3 gene product. FEBS Lett. 342:23-28.
20. Gomez, M.J., L.R. Desviat, R. Merchante, and J.A. Ayala. 1993. Involvement of the $\mathrm{NH}_{2}-$ and $\mathrm{COOH}$-terminal ends of PBP3 of Escherichia coli on $\beta$-lactam binding, membrane localization and function of the protein, pp. 309-318. In M.A. de Pedro, J.V. Höltje, and W. Löffelhardt (eds.), Bacterial growth and lysis: Metabolism and structure of the bacterial sacculus. Plenum Press, New York.

21. Granier, B., M. Jamin, M. Adam, M. Galleni, B. Lakaye, W. Zorzi, J. Grandchamps, J.M. Wilkin, C. Fraipont, B. Joris, C. Duez, M. Nguyen-Distèche, J. Coyette, M. Leyh-Bouille, J. Dusart, L. Christiaens, J.M. Frère, and J.M. Ghuysen. 1994. Serine-type D-Ala-D-Ala peptidases and penicillin-binding proteins. Methods Enzymol. 244:249-266.

22. Hanaki, H., H. Akagi, Y. Masaru, T. Otani, A. Hyodo, and K. Hiramatsu. 1995. Toc-39, a novel parenteral broad-spectrum cephalosporin with excellent activity against methicillin-resistant Staphylococcus aureus. Antimicrob. Agents Chemother. 39:1120-1126.

23. Hakenbeck, R. 1995. Target-mediated resistance to $\beta$-lactam antibiotics. Biochem. Pharmacol. 50:1121-1127.

24. Hellingwerf, K.J., P.W. Postma, J. Tommassen, and H.V. Westerhoff. 1995. Signal transduction in bacteria: Phospho-neural network(s) in Escherichia coli? FEMS Microbiol. Rev. 16:309-321.

25. Höltje, J.V. 1995. From growth to autolysis: The murein hydrolases in Escherichia coli. Arch. Microbiol. 164:243-254.

26. Jacobs, C., L. Huang, E. Bartowsky, S. Normark, and J.T. Park. 1994. Bacterial cell wall recycling provides cytosolic muropeptides as effectors for $\beta$-lactamase induction. EMBO J. 13:4684-4694.

27. Jacobs, C., B. Joris, M. Jamin, K. Klarsov, J. Van Beeumen, D. Mengin-Lecreulx, J. van Heijenoort, J.T. Park, S. Normark, and J.M. Frère. 1995. AmpD, essential for both $\beta$-lactamase regulation and cell wall recycling, is a novel cytosolic $\mathrm{N}$-acetylmuramyl-L-alanine amidase. Mol. Microbiol. 15:553-559.

28. Jamin, M., C. Damblon, S. Millier, R. Hakenbeck, and J.M. Frère. 1993. Penicillin-binding protein $2 \times$ of Streptococcus pneumoniae: Enzymic activities and interactions with $\beta$-lactams. Biochem. J. 292:735-741.

29. Joris, B., K. Hardt, and J.M. Ghuysen. 1994. Induction of $\beta$-lactamase and low-affinity penicillin-binding protein $2^{\prime}$ synthesis in Gram-positive bacteria, pp. 505-516. In New comprehensive biochemistry. Bacterial cell wall, Vol. 27. Elsevier Science Publishers, Amsterdam.

30. Joseleau-Petit, D., D. Thévenet, and R. D'Ari. 1994. ppGpp concentration, growth without PBP2 activity, and growth rate control in Escherichia coli. Mol Microbiol. 13:911-917.

31. Nicholas, R.A., D.R. Lamson, and D.E. Schultz. 1993. Penicillinbinding protein $1 \mathrm{~B}$ from Escherichia coli contains a membrane association site in addition to its transmembrane anchor. J. Biol. Chem. 268:5632-5641.

32. Orengo, C.A., D.T. Jones, and J.M. Thornton. 1994. Protein superfamilies and domain superfolds. Nature (London) 372:631-634.

33. Piras, G., D. Raze, A. El Kharroubi, D. Hastir, S. Englebert, J. Coyette, and J.M. Ghuysen. 1993. Cloning and sequencing of the low affinity penicillin-binding protein $3 r$-encoding gene of Enterococcus hirae S185. Modular design and structural organization of the protein. J. Bacteriol. 175:2844-2852.

34. Popham, D.L., and P. Setlow. 1993. Cloning, nucleotide sequence, and regulation of the Bacillus subtilis $p b p F$ gene, which codes for a putative class A high-molecular-weight penicillin-binding protein. J. Bacteriol. 175:4970-4876.

35. Sanchez, M., A. Valencia, M.J. Ferrandiz, C. Sander, and M. Vicente. 1994. Correlation between the structure and biochemical activities of FtsA, an essential cell division protein of the actin family. EMBO J. 13:4919-4925.

36. Severin, A. M.V. Vaz Pato, A.M. Sa Figueiredo, and A. Tomasz. 1995. Drastic changes in the peptidoglycan composition of peni- 
cillin resistant laboratory mutants of Streptococcus pneumoniae. FEMS Microbiol. Lett. 130:31-35.

37. Shlaes, D.M., and L.B. Rice. 1994. Bacterial resistance to the cyclic glycopeptides. Trends Microbiol. 2:3885-389.

38. Smith, A.M., and K.P. Klugman. 1995. Alterations in penicillinbinding protein $2 \mathrm{~B}$ from penicillin-resistant wild-type strains of Streptococcus pneumoniae. Antimicrob. Agents Chemother. 39:859-867.

39. Wang, $X$., and J. Lutkenhaus. 1993. The FtsZ protein of Bacillus subtilis is localized at the division site and has GTPase activity that is dependent upon FtsZ concentration. Mol Microbiol. 9:435-442.

40. Wu, C.Y.E., J. Hoskins, L.C. Blaszczak, D.A. Preston, and P.L. Skatrud. 1992. Construction of a water-soluble form of penicillinbinding protein 2a from a methicillin-resistant Staphylococcus aureus isolate. Antimicrob. Agents Chemother. 36:533-539.
41. Wu, C.Y.E., W.E. Alborn, Jr., J.E. Flokowitsch, J. Hoskins, S. Unal, L.C. Blaszczak, D.A. Preston, and P.L. Skatrud. 1994. Site-directed mutagenesis of the mecA gene from a methicillin-resistant strain of Staphylococcus aureus. J. Bacteriol. 176:443-449.

42. Zijderveld, C.A., M.E.G. Aarsman, and N. Nanninga. 1995. Differences between inner membrane and peptidoglycan-associated PBP1B dimers of Escherichia coli. J. Bacteriol. 177:1860-1863.

Address reprint requests to: Jean-Marie Ghuysen Centre d'Ingénierie des Protéines Université de Liège Institut de Chimie, B6 B-4000 Sart Tilman (Liège 1), Belgium 
This article has been cited by:

1. Sampath C Abeylath, Edward Turos. 2008. Drug delivery approaches to overcome bacterial resistance to $\beta$-lactam antibiotics. Expert Opinion on Drug Delivery 5:9, 931-949. [CrossRef]

2. SHANGWEI WU, CLAUDIA PISCITELLI, HERMINIA de LENCASTRE, ALEXANDER TOMASZ. 1996. Tracking the Evolutionary Origin of the Methicillin Resistance Gene: Cloning and Sequencing of a Homologue of mecA from a Methicillin Susceptible Strain of Staphylococcus sciuriTracking the Evolutionary Origin of the Methicillin Resistance Gene: Cloning and Sequencing of a Homologue of mecA from a Methicillin Susceptible Strain of Staphylococcus sciuri. Microbial Drug Resistance 2:4, 435-441. [Abstract] [PDF] [PDF Plus]

3. Kevin D YoungPeptidoglycan . [CrossRef] 